\title{
Accurate Measurements of High Strain Gradients near Notches Using a Feature-Based DIC Algorithm
}

\author{
Giancarlo L. G. Gonzáles ${ }^{1}$, Leonardo D. Rodrigues ${ }^{2}$ and José L. F. Freire ${ }^{1}$ \\ 1. Department of Mechanical Engineering, Pontifical Catholic University of Rio de Janeiro, Rua Marquês de São Vicente 225, \\ Gávea, Rio de Janeiro RJ-22543-900, Brazil \\ 2. Department of Mechanical Engineering, UFPA, Rua Augusto Corrêa, 1 - Guamá, Belém-PA 66075-110, Brazil
}

\begin{abstract}
In this work, the efficiency of a feature-based DIC (digital image correlation) algorithm for measuring high strain gradients was investigated by means of numerical and actual experiments. The so-called SIFT-Meshless method consisted of a novel formulation involving the SIFT (scale-invariant feature transform) feature detector with a self-adaptive meshless formulation. Whereas the numerical experiments aimed to evaluate the accuracy and the spatial resolution, the actual experiments aimed to demonstrate in practice the above findings. A stereoscopic system and a micro-stereoscopic system were used to perform high strain gradient measurements in notched specimens of different materials and notch sizes. This paper concludes that the feature-based algorithm is able to provide accurate strain measurements at high strain gradient regions, even under conditions of plasticity. Moreover, the algorithm showed its efficiency to capture the peak strain near the notch boundary. Lastly, a spatial resolution study proposes a link between the desired accuracy and the pixel resolution required to perform accurate measurements of high strain gradients.
\end{abstract}

Key words: High strain gradients, digital image correlation, SIFT, meshless.

\section{Introduction}

Regions of high strain gradients (i.e., large change in strain values within a small distance) are commonly observed surrounding stress concentrators, such as holes, shoulder fillets, and regions generally called shallow or deep notches. Therefore, their characterization requires of experimental techniques with high spatial resolution to capture the peak strain expected near the notch boundary, which is an area of great interest in fatigue analysis. In that field, the DIC (digital image correlation) [1-4] is presently the most employed technique by researchers. If the standard subset-based DIC is used [5-8], accurate strain measurements near the notch boundary are difficult to obtain because the point of measurement is considered by default at the center of the subset, therefore, the information at the boundary of the region of interest

Corresponding author: José L. F. Freire, associate professor, Ph.D., research fields: structural integrity and experimental stress analysis. (i.e., the notch boundary) is absent. Moreover, the measured gradient can be more flattened than the real one, depending on the DIC parameters used in the analysis.

In recent years, alternative algorithms [9-12] have been proposed to optimize the standard DIC in order to ensure more accurate solutions when non-homogenous strain field mesurements are required. Another recently proposed algorithm is the SIFT-Meshless method [13] which uses a feature-based matching approach for correlating images. Features are points or small patches on the image that differ from their immediate surrounding region and can easily be extracted by means of efficient algorithms. The SIFT-Meshless algorithm adopts the SIFT [14, 15] which is a robust local feature extractor with outstanding performance. By using SIFT, displacement measurements are obtained by tracking the successfully matched features from the reference to the deformed image. Then, a meshless 
formulation with self-adaptive domain size is applied for solving the displacement field and its derivatives (more information about the construction of meshless shape functions and their properties can be found in Ref. [16]). The self-adaptive meshless formulation improves the accuracy of the measurements when low and high strain gradient fields co-exist in the same analysis. Moreover, the method is capable to obtain strain data at the notch boundary, since feature points are extracted from this region.

In this paper, the SIFT-Meshless method is used to perform high strain gradient measurements around shallow and deep U-notches. Two applications using different materials and different types of loading are presented: the elastic problem of deep U-notches in two polycarbonate specimens under uniaxial tensile load; and the elastic-plastic problem of a semicircular notch in a stainless steel specimen under bending load. In addition, a subset-based DIC analysis was performed by using the commercially available VIC-2D and VIC-3D systems from Correlated Solution Inc [17]. Moreover, the experiments were simulated using the finite element ANSYS software to provide an additional source of verification.

Lastly, the spatial resolution and accuracy of the two DIC algorithms were investigated by means of numerical experiments. The spatial resolution is defined in Refs. $[11,18]$ as the minimum spatial unit between two independent data points required to perform a measurement. Based on this concept, the spatial resolution for the subset-based DIC method is determined by the subset size, which defines the distance between two adjacent measurement points in the correlation process. However, this definition is not applicable to non-traditional DIC algorithms, such as the SIFT-Meshless method. From the literature review [11], one way to evaluate the spatial resolution for non-traditional DIC algorithms is by using simulated images undergoing sinusoidal displacements with various amplitudes and spatial frequencies. In the present study, not only a sinusoidal function but also an analytical stress-concentration displacement fields were considered to simulate different cases of strain gradients. The numerical experiments aimed to establish a methodology to evaluate the accuracy and spatial resolution of traditional and non-traditional DIC algorithms for high strain gradient measurements. Moreover, the final objective is to present a simple procedure to obtain the expected error when measuring the actual maximum strain at the notch boundary for a given optical configuration, where the field magnification and consequently pixel resolution are known beforehand.

\section{Fundamentals of the Local-based SIFT-Meshless Method}

\subsection{Displacement Measurements Using SIFT}

The SIFT technique is used to measure relative displacement of features successfully matched in two images of the specimen's surface - one captured before and the other after deformation (Fig. 1). The tracking feature procedure involves three steps: feature detection, feature description, and feature matching. These are briefly described as follows (more detailed information about the SIFT algorithm can be found in Refs. [14, 15]).

The feature detection step consists in searching for distinctive and representative feature points. The cost of extracting the SIFT features is minimizing by using a cascade filtering approach in which the more expensive operations are applied only at locations that pass all prior tests. During this stage, features sensitive to noise and poorly localized features are discarded.

In the feature description step, a numerical vector called feature descriptor is built based on the image properties of a support region around each feature point, as shown in Fig. 2. The image properties include gradient magnitude, gradient direction, and pixel intensity. The final feature descriptor is a vector with 128 elements describing the unique properties of the corresponding feature point and its support region. 


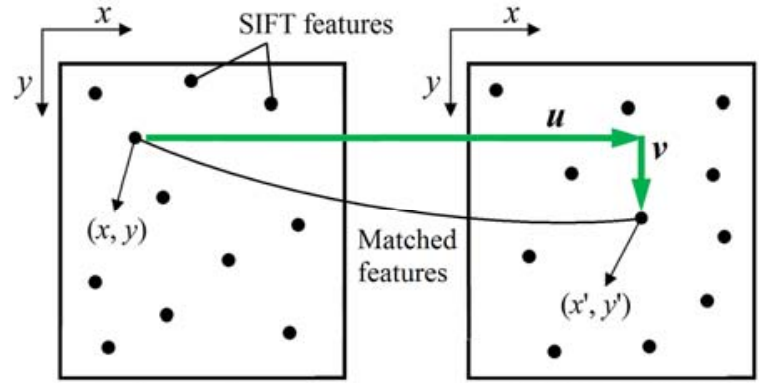

Reference Image

Deformed Image

Fig. 1 Displacement measurements obtained by matching SIFT features.

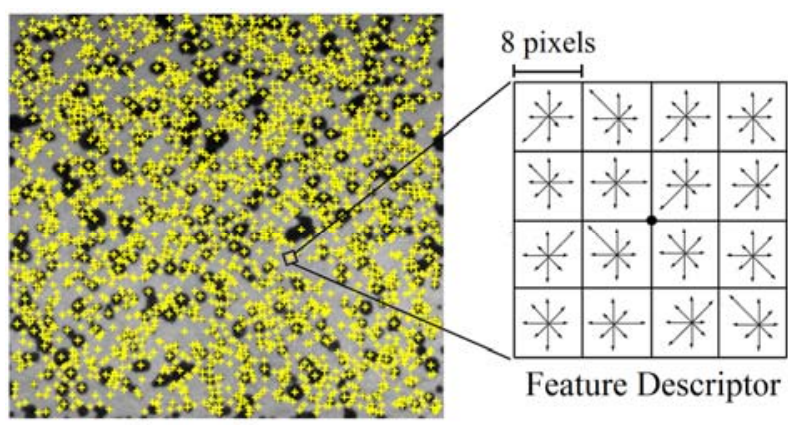

Feature Detector

Fig. 2 Example of SIFT features detected on a dotted image test (left), and feature identification given by its descriptor (right).

In the feature matching step, correspondence features between images are computed based on the similarity of their descriptors. To do so, the Euclidean distance is the commonly used method for matching features in the SIFT algorithm. The best match for each descriptor is found by minimizing the Euclidean distance. In order to discard poorly or overly ambiguous matched features, the distance ratio between the closest distance and the second-closest distance is evaluated. The SIFT-Meshless method uses a distance ratio of 0.3-0.4 in order to reduce the possibility of false matches, due to the large number of points extracted from images. Moreover, since a stereovision system is used, the SIFT features are also used to determine corresponding features in the stereo images for recovering the 3-D positions of the images captured by the cameras using a stereo triangulation algorithm.

\subsection{Meshless Formulation}

Once the SIFT features are located in the undeformed-deformed pair of images, a meshless formulation based on the Moving Least Squares approximation [19] is used for solving the displacement and strain field.

Thus, the meshless approximation for the $u$-component of the displacement field can be written,

$$
u^{h}(\mathbf{x})=\sum_{i}^{n} \phi_{i}(\mathbf{x}) u_{i}=\Phi(\mathbf{x}) \mathbf{U}
$$

where $n$ is the number of nodes (SIFT features) in the support domain of any point $\mathbf{x}=[x, y]^{\mathrm{T}}, \boldsymbol{\Phi}(\mathrm{x})$ is the matrix of the shape function values of the $i$-th node, and $\mathbf{U}$ is the vector of the nodal displacements provided by tracking the SIFT features.

The support domain determines the number of nodes to be used to support or approximate the function value at a specific point of evaluation. In addition, the support domain can be weighted using a weight function, which gives more influence to points close to the evaluation point. The authors tested several weight functions available in the literature, and similar results were obtained. The SIFT-meshless formulation adopted the exponential weight function.

$$
w(r)=\left\{\begin{array}{cc}
e^{-(r / 0.4)^{2}}, & r \leq 1 \\
0, & r>1
\end{array}\right.
$$

In Eq. (2), the value of the weight function decreases with the distance $r$ from the center of the function, i.e., points near to the evaluation point have more influence on the value of the function than those points far away. The dimension of $r$ is calculated using the equation:

$$
r=\frac{\left\|\mathbf{x}-\mathbf{x}_{i}\right\|}{d m_{i}}
$$

In this equation, $d m_{i}$ is the dimension of the influence domain determined by

$$
d m_{i}=\alpha_{s} \cdot d_{i}
$$

where, $d_{i}$ is the radius of the domain, and $\alpha_{s}$ is a scaling parameter, both initially prescribed by the analyst.

As shown in Fig. 3, the support domain never extends beyond the periphery of the problem domain. 


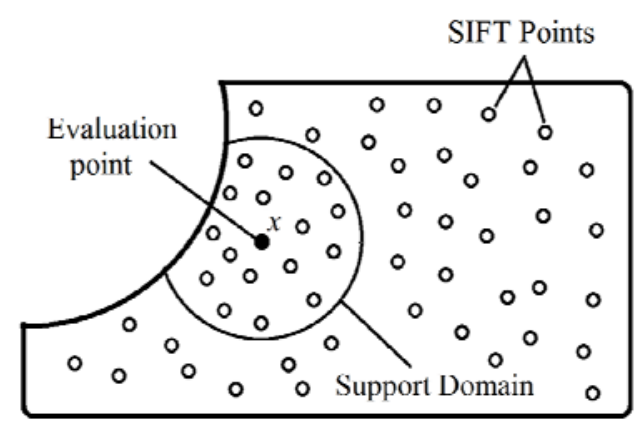

Fig. 3 Example of the support domain for the node $x$.

For domains with irregularly distributed nodes (such as the features points provided by SIFT), the use of an influence domain works well to select nodes for constructing shape functions.

\subsubsection{Moving Least Squares Approximation}

The MLS shape function $\phi_{i}$ associated with node $x_{i}$ can be calculated as

$$
\phi_{i}(\mathbf{x})=\sum_{j}^{m} p_{j}(\mathbf{x})\left(\mathbf{A}^{-1}(\mathbf{x}) \mathbf{B}(\mathbf{x})\right)_{j i}=\mathbf{P}^{\mathrm{T}} \mathbf{A}^{-1} \mathbf{B}_{i}
$$

where, $\mathbf{P}^{\mathrm{T}}$ is a polynomial basis of order $m$ (e.g., linear, quadratic).

The matrices $\mathbf{A}$ and $\mathbf{B}$ are defined by

$$
\begin{gathered}
\mathbf{A}(\mathbf{x})=\sum_{i=1}^{n} w\left(\mathbf{x}-\mathbf{x}_{i}\right) \mathbf{P}\left(\mathbf{x}_{i}\right) \mathbf{P}^{\mathrm{T}}\left(\mathbf{x}_{i}\right) \\
\mathbf{B}(\mathbf{x})=\left[\begin{array}{lll}
w\left(\mathbf{x}-\mathbf{x}_{1}\right) \mathbf{P}^{\mathrm{T}}\left(\mathbf{x}_{1}\right) & \ldots & \left.w\left(\mathbf{x}-\mathbf{x}_{n}\right) \mathbf{P}^{\mathrm{T}}\left(\mathbf{x}_{n}\right)\right]_{i}
\end{array}\right.
\end{gathered}
$$

From Eq. (6), one can see that the minimum number of nodes needed for matrix $\mathrm{A}$ to be regular depends on the polynomial basis function used. In this work, a quadratic basis function was adopted.

The expression in Eq. (1) can also be used for the displacement component $v(\mathbf{x})$. Thus, the displacement field in the deformed surface is defined as

$$
\left\{\begin{array}{l}
u \\
v
\end{array}\right\}^{h}=\sum_{i}^{n}\left[\begin{array}{cc}
\phi_{i} & 0 \\
0 & \phi_{i}
\end{array}\right]\left\{\begin{array}{l}
u_{i} \\
v_{i}
\end{array}\right\}
$$

The strains at any point in the problem domain are obtained in terms of the nodal displacement components by using the strain-displacement relation defined by

$$
\left\{\begin{array}{c}
\varepsilon_{x} \\
\varepsilon_{y} \\
\varepsilon_{x y}
\end{array}\right\}^{h}=\left[\begin{array}{cc}
\partial / \partial x & 0 \\
0 & \partial / \partial y \\
\partial / \partial y & \partial / \partial x
\end{array}\right]\left\{\begin{array}{l}
u \\
v
\end{array}\right\}^{h}=\sum_{i}^{n}\left[\begin{array}{cc}
\phi_{i, x} & 0 \\
0 & \phi_{i, y} \\
\phi_{i, y} & \phi_{i, x}
\end{array}\right]\left\{\begin{array}{c}
u_{i} \\
v_{i}
\end{array}\right\}
$$

In this way, the first order partial derivatives of the MLS shape with respect to $x$ and $y$ direction are required, which can be easily computed by differentiation of Eq. (5). It is worth mentioning that the small-strain approximation defined in Eq. (9) is used for strains less than $1 \%$. When needed, other strain definitions (e.g. large strains) can be used.

\subsubsection{Self-adaptive Domain Process}

It was verified that high strain gradients require small influence domains to perform accurate approximations. On the other hand, large influence domains provide a smoothed solution, which is most appropriate for characterizing uniform strain distributions. Moreover, according to Eq. (4), the dimension of the influence domain is determined by the scaling parameter $\alpha_{s}$. In this way, the self-adaptive process consists in determining a suitable value for $\alpha_{s}$ for each evaluation point according to the strain-gradient behavior. For that, the proposed test function shown in Fig. $4 \mathrm{~b}$ is used. This function relates the strain-gradient behavior with an appropriate value for $\alpha_{s}$. To obtain an approximation of the strain-gradient behavior in the analyzed region, a first meshless approximation is calculated with a high value for $\alpha_{s}$, such as 3 or 4 . Then, the gradient of this first approximation is computed in the direction of the notch tip (e.g., along the $x$-direction of the notch shown in Fig. 4a). The magnitude of this gradient shows how fast the strain rises in that direction, indicating areas of high strain concentration. Lastly, by using the test function in Fig. $4 b$, the approximations are re-computed with the new values for $\alpha_{s}$, and the corresponding strain components on the surface are obtained.

\section{Experimental Details}

Two data acquisition systems were used: a 

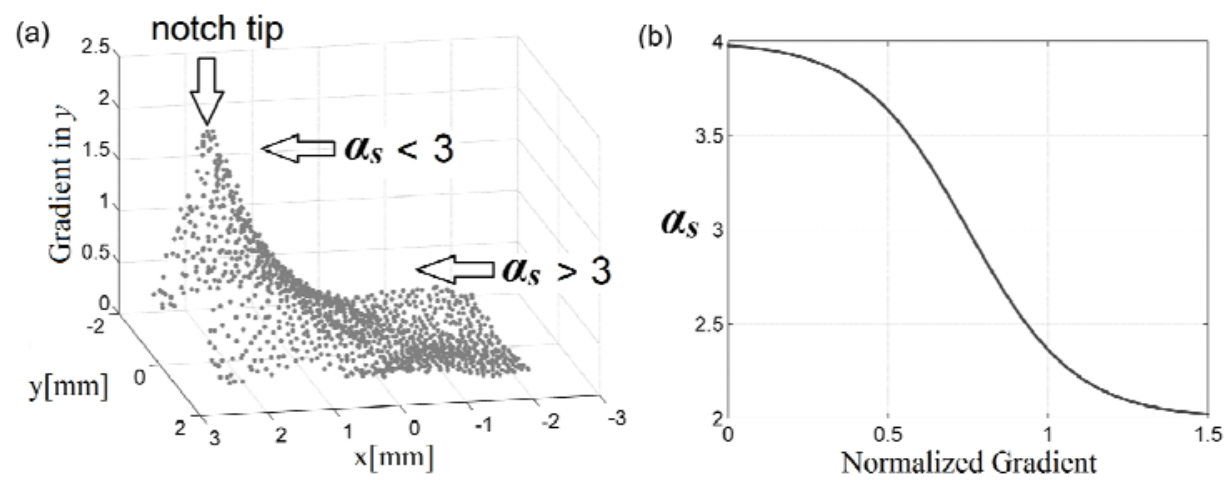

Fig. 4 (a) Example of gradient behavior calculated for the region ahead of the notch (b) test function used for calculating suitable values for $\alpha_{s}$.

Table 1 Experimental matrix.

\begin{tabular}{llllll}
\hline Specimen & Notch radius & Material & Analysis & Test & Optics System \\
\hline PC-1 & $2.4 \mathrm{~mm}$ & Poly-carbonate & Elastic & Tensile & Micro \\
PC-2 & $1.0 \mathrm{~mm}$ & Poly-carbonate & Elastic & Tensile & Micro \\
SS-3 & $12.5 \mathrm{~mm}$ & Stainless steel & Elastic-plastic & Bending & HM lenses \\
\hline
\end{tabular}

stereoscopic system with HM (high magnification) lenses for measuring areas with side-lengths ranging from $5 \mathrm{~mm}$ to $50 \mathrm{~mm}$, and a micro-stereoscopic (micro) system for measuring areas with side-lengths ranging from $1 \mathrm{~mm}$ to $7 \mathrm{~mm}$. The digital CCD (charge-coupled device) cameras had resolutions of $2,448 \times 2,048$ pixels. Table 1 gives the experimental matrix.

Calibrating stereovision systems was completed in the calibration module of the VIC-3D software. The same calibration parameters were used for both DIC algorithms to exclude possible sources of errors.

The sample surfaces were covered with a uniform coat of white paint, after which black dots were added. The size and distribution of such dots is related to the optical system used. For the macroscopic length scales, the random black-and-white pattern was performed with spray painting. For the microscopic length scales, small sizes of dots were obtained with an airbrush that provided a smooth transition from fine to medium spraying. The same speckle pattern was used for both algorithms. In the traditional DIC approach, the surface preparation aims to create a random high contrast pattern on the specimen's surface. In the SIFT-Meshless method, the objective is to provide a large number of potential candidates to be feature points that can be detected by the SIFT algorithm.

\section{Strain Concentration in Deep U-Notch Specimens under Linear-Elastic Tensile Load}

\subsection{Experimental Conditions}

The experimental test performed in this section aimed to obtain the high strain gradient distribution ahead of a sharp notch subjected to tensile stresses. Two specimens made of polycarbonate with $3 \mathrm{~mm}$ thickness containing central deep U-notches with radii of $2.4 \mathrm{~mm}$ (PC-1) and $1 \mathrm{~mm}$ (PC-2) were used. The geometry of the samples and the experimental set-up are shown in Fig. 5. The mechanical properties of the polycarbonate, namely Modulus of Elasticity and Poisson's ratio are respectively $2.3 \mathrm{MPa}$ and 0.4 . The experimental setup used the micro-stereoscopic system. The field of view was about $6.1 \times 5.1 \mathrm{~mm}^{2}$ surrounding the notch tip with pixel resolution of approximately $2.5 \mu \mathrm{m}$ on the object plane for both PC-1 and PC-2 specimens. The loads were applied to the specimens by means of a force application system into which a load cell device was installed. Thus, successive tensile loadings of $25,50,100 \mathrm{~N}$ were 


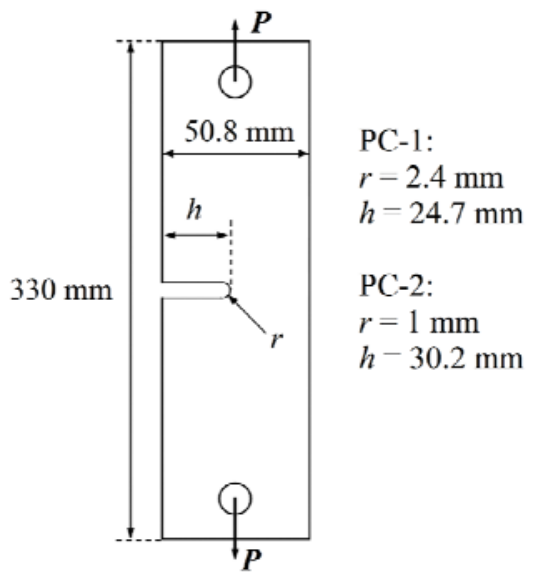

Fig. 5 Geometry of the polycarbonate specimens.

applied to PC-1, and of $15,30,60 \mathrm{~N}$ to PC-2. One image was captured at each load increment.

\subsection{Results}

Surface strains around the notch were assessed with the VIC-3D software using subset sizes of 43 pixels and 49 pixels for PC-1 and PC-2, respectively. For both analyses, the step size was set to 11 pixels and strain windows of 15 . In the SIFT-Meshless analysis, approximately 6,500 and 5,900 feature points were successfully matched in the PC-1 and PC-2 captured images, respectively. The minimum domain $\left(d_{i}\right)$ was set to 200 pixels for both analyses.

Fig. 6 depicts the strain distribution determined by the VIC-3D and SIFT-Meshless algorithms along the $x$-axis $(y=0)$ at the different loading stages. These strain values were compared with results determined by the FEM simulation using Ansys (3-D FEM analysis, tetrahedral elements, Solid-187).

From these test results, it can be seen that the experimental measurements and the numerical results showed good agreement. Moreover, the high strain gradient regions can be clearly identified being that it was more severe in PC-2, where, for the maximum loading of $60 \mathrm{~N}$, the strain value decreased drastically from $0.2 \%(2,000 \mu \varepsilon)$ to $0.7 \%(7,000 \mu \varepsilon)$ in a distance of $1 \mathrm{~mm}$ ahead of the notch tip. Notice that the maximum strains at the notch tip were successfully quantified by the SIFT-Meshless method. In the (a)

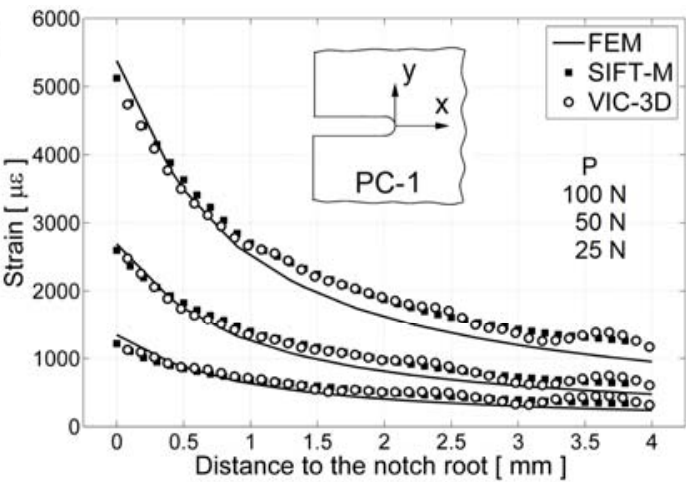

(b)

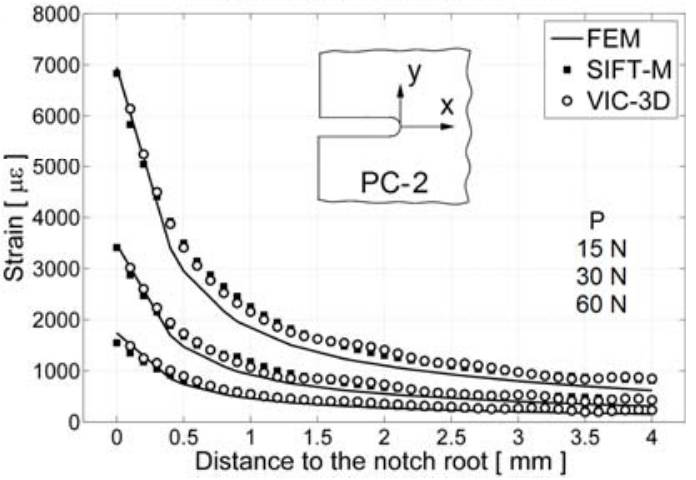

Fig. 6 Comparison between numerical and experimental strain distributions extracted from the strain component $\varepsilon_{y y}$ along the $x$-axis (a) PC-1 specimen (b) PC-2 specimen.

VIC-3D analysis, the nearest point of measurement from the notch was located at about $75 \mu \mathrm{m}$ and $70 \mu \mathrm{m}$ for PC-1 and PC-2, respectively. In addition, Fig. 7 depicts the strain fields for the deformed state measured in PC-1 at $100 \mathrm{~N}$ by the two DIC algorithms.

\section{Strain Concentration in Circumferential Notch Specimen under Elastic-Plastic Bending}

\subsection{Experimental Conditions}

The experiment performed in this section aimed to induce a plastic strain-gradient near the notch tip by four-point bending test. Therefore, it is required that the algorithm be able to quantify the strain-gradient and the nonlinear behavior of the material ahead of the notch tip after the yield stress (or elastic limit) is reached, generally at $0.2 \%$ strain (i.e., $2,000 \mu \varepsilon$ ).

The bending test was performed in a servo-hydraulic Instron machine equipped with a four-point bending 
(a)

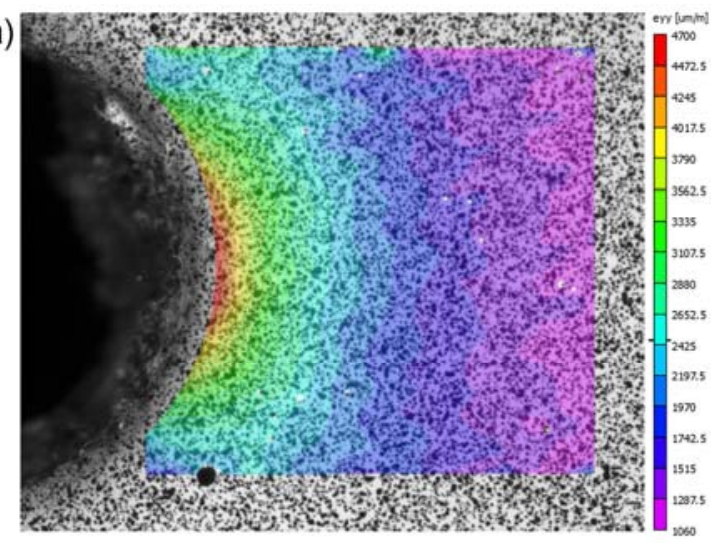

(b)

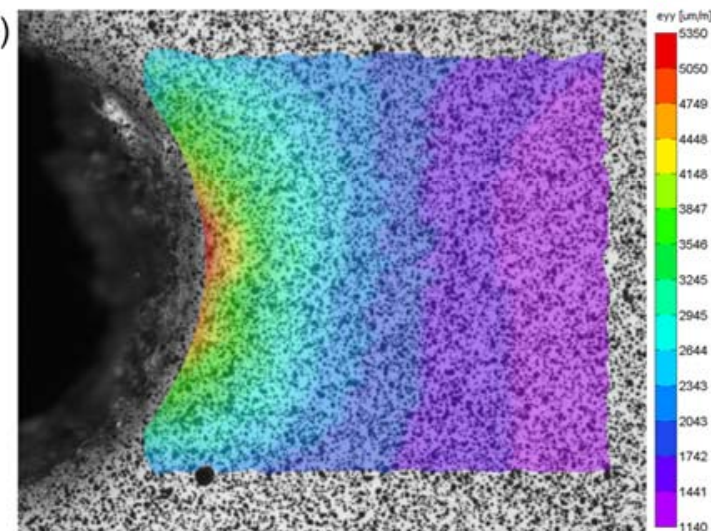

Fig. 7 Full-field distribution for the strain component $\varepsilon_{y y}$ at $100 \mathrm{~N}$ (a) VIC-3D (b) SIFT-Meshless method.

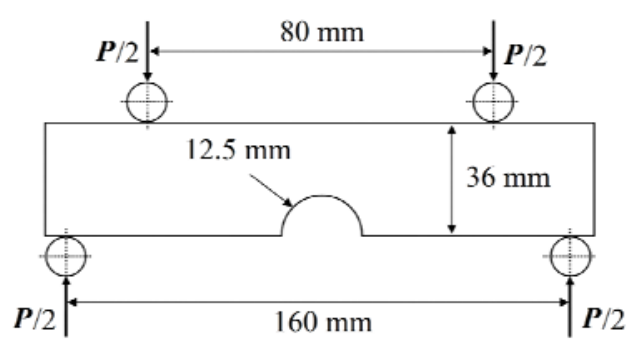

Fig. 8 Sketch of the experimental setup of the 4-point bending.

fixture. The specimen was a 304 stainless-steel plate with thickness of $5 \mathrm{~mm}$ and a semicircular notch with radius of $12.5 \mathrm{~mm}$. The specimen geometry and the four-point bending setup are shown schematically in Fig. 8. The mechanical properties of the stainless-steel were determined experimentally: Modulus of Elasticity $(\mathrm{E})=195 \mathrm{MPa}$ and the yield stress $\mathrm{S}_{\mathrm{y}}, 0.2 \%$ $=250 \mathrm{MPa}$.

In this experiment, the stereoscopic system equipped with high magnification lenses was used. The cameras were placed in front the specimen resulting in a field of view of about $35 \times 30 \mathrm{~mm}^{2}$ with a pixel resolution of approximately $14 \mu \mathrm{m}$ on the object plane. Images were taken from the specimen with loadings of 4, 6, 8 and $10 \mathrm{kN}$, whereby the elastic limit was attained and plastic strains at the notch tip were induced.

\subsection{Results}

After the images were captured, they were processed using the VIC-3D software with a subset size of 25 pixels, step size of 8 pixels, and strain windows of 15 . In the SIFT-Meshless analysis, approximately 8,500 feature points were successfully matched between images, and the minimum domain used was set to approximately 180 pixels. The elastic-plastic analysis for the 4-point bending problem was simulated using Ansys software (3-D FEM analysis, tetrahedral elements, Solid-187). The numerical solution used an isotropic multilinear model, described by the plastic stress-strain curve of the 304 stainless-steel.

The maximum strain values obtained by the FEM simulation and measured by the two experimental techniques are plotted in Fig. 9. The inclination of the curve indicates the increment of the plasticity at the notch tip. It can be seen that significant plastic strain was induced after the load of $6 \mathrm{kN}$.

Fig. 10 shows the axial strain values determined by the VIC-3D and SIFT-Meshless methods along the $y$-axis $(x=0)$ for the loadings of $8 \mathrm{kN}$ and $10 \mathrm{kN}$. The strain distributions obtained by the two DIC algorithms were in good agreement with the finite

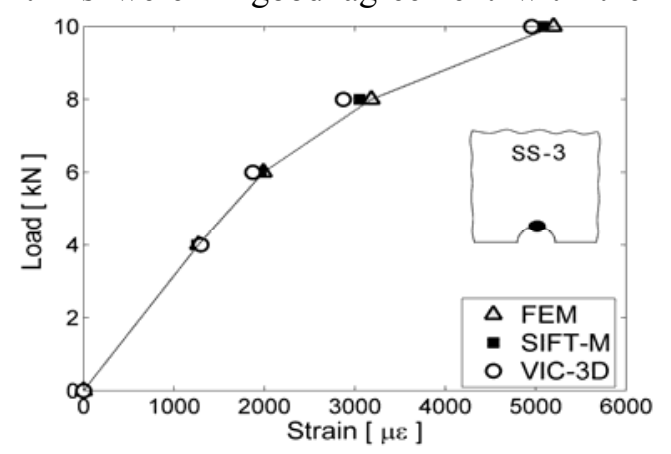

Fig. 9 Maximum strain at notch tip in SS-3 specimen. 
(a)

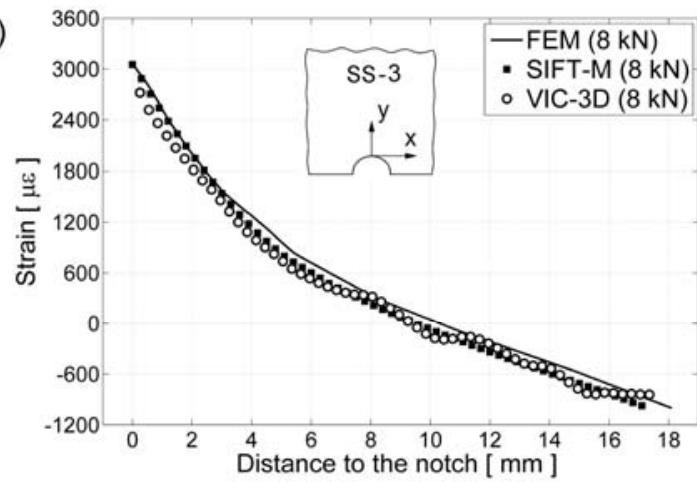

(b)

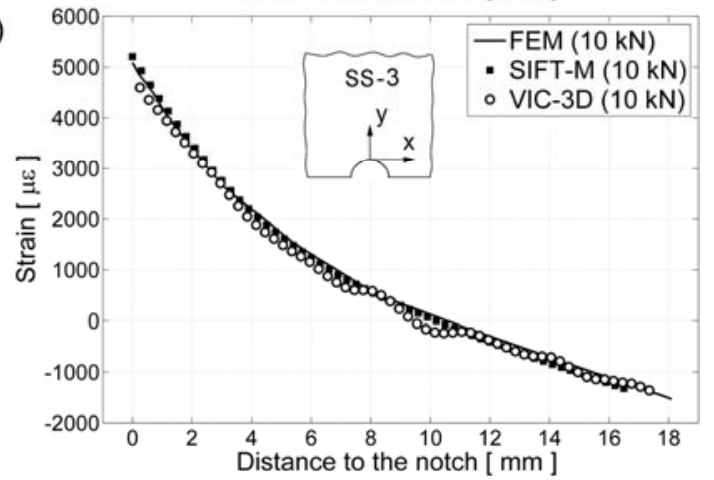

Fig. 10 Comparison between numerical and experimental strain distributions extracted from the strain component $\varepsilon_{x x}$ along the selected path (a) $8 \mathrm{kN}$ (b) $10 \mathrm{kN}$.

element simulations using the Ansys software, even when significant plastic strain has been reached in the area surrounding the notch. In addition, strain measurements at the notch boundary were successfully obtained using the SIFT-Meshless method, where the maximum plastic strain was approximately $0.5 \%$ when the test load was $10 \mathrm{kN}$. In the VIC-3D analysis, the point nearest to the notch was located at $250 \mu \mathrm{m}$ from the notch boundary. It can be also observed in Fig. 11, where the strain fields for the deformed state measured in SS-3 at $10 \mathrm{kN}$ by the two DIC algorithms are shown. Moreover, it was noticed that the SIFT-Meshless algorithm adapts the calculation of the full-field solution to the presence of high and low strain gradients by decreasing or increasing the influence domain at each evaluated feature point.

\section{Accuracy and Spatial Resolution in High Strain Gradient Measurements}

The spatial resolution study was focused on the (a)

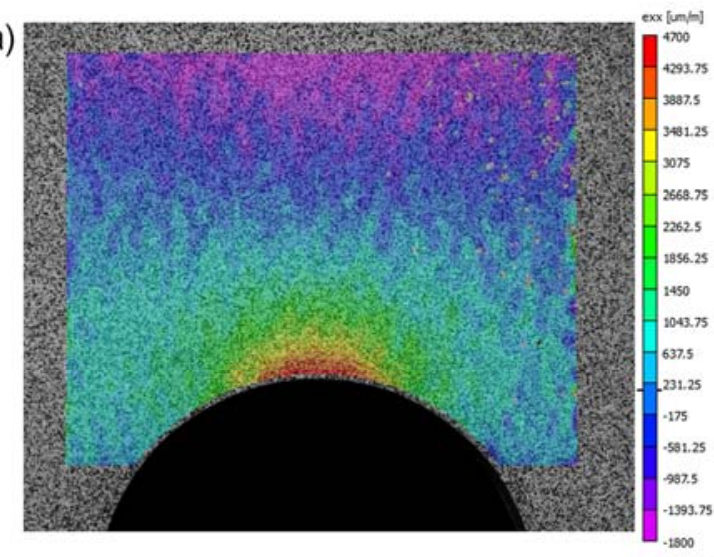

(b)

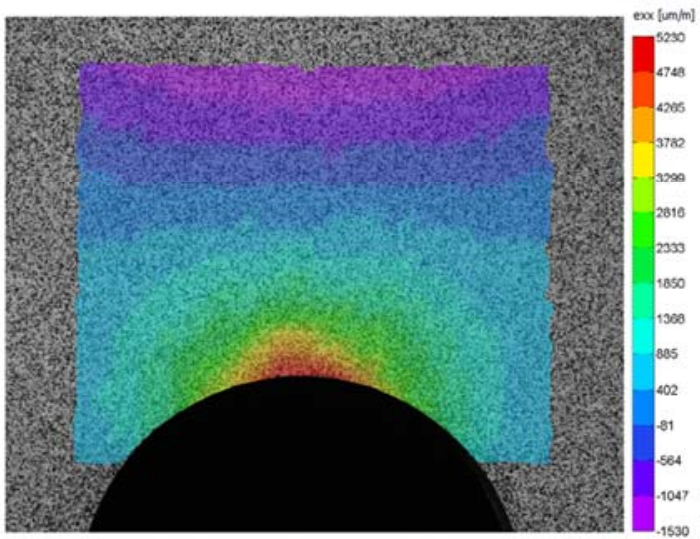

Fig. 11 Full-field distribution for the strain component $\varepsilon_{x x}$ at $10 \mathrm{kN}$ (a) VIC-3D (b) SIFT-Meshless method.

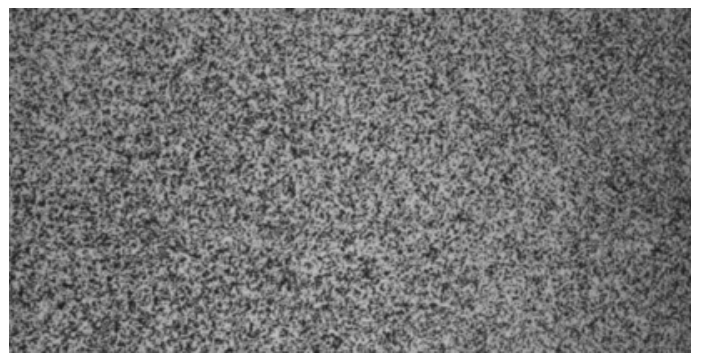

Fig. 12 Speckle pattern used for numerical test.

assessment of the strain error, whereas that, strain is mainly the final desired quantity to be measured. For that, different numerical tests were carried out using numerically deformed images by imposing unidirectional in-plane deformation fields with highly heterogeneous behavior.

Fig. 12 shows the speckle pattern used as reference image $(1,200 \times 800$ pixels $)$ which was cropped from an actual experimental image (8-bit, 2,248 $\times 2,048$ pixels). The deformed images were generated using the Matlab ${ }^{\circledR}$ and its Image Processing Toolbox ${ }^{\mathrm{TM}}$. 
The present analysis assumed displacement field functions imposed along the horizontal direction with zero displacement in the vertical direction. The error assessment was based on the measurement accuracy to capture the maximum peak strain for the different configurations of imposed strain gradients. Since the synthetic displacement field is unidirectional ( $x$-direction), the corresponding imposed strain gradient is the same for all vertical line (column) of the image. Therefore, the strains measured for all points located over any column can be statistically analyzed and compared with the imposed strain value to the considered column. Thus, the differences between the measured and imposed strains can be used in order to predict the expected AL (amplitude loss) for each DIC algorithm evaluated. For that, the following model was used:

$$
A L=\frac{\left|\varepsilon_{\text {imposed }}^{\max }-\mu_{\text {smax }}\right|+3 \cdot \sigma_{\text {smax }}}{\varepsilon_{\text {imposed }}^{\max }} \cdot 100
$$

In this model, the 3-sigma indicates a $99.8 \%$ of certainty on amplitude determination. The arithmetic mean $(\mu)$ and the standard deviation $(\sigma)$ are defined as:

$$
\begin{gathered}
\mu_{\varepsilon \max }=\frac{\sum \varepsilon_{\max }^{\text {measured }}}{n} \\
\sigma_{\text {Emax }}=\sqrt{\frac{1}{n-1} \sum\left(\varepsilon_{\text {max }}^{\text {masured }}-\mu_{\text {Emax }}\right)^{2}}
\end{gathered}
$$

Finally, the error associated with the whole field strain measurement in terms of the RMSE (root mean square error) is calculated by

$$
R M S E=\sqrt{\frac{1}{n} \sum\left(\varepsilon_{\text {imposed }}(x, y)-\varepsilon_{\text {measured }}(x, y)\right)^{2}}
$$

where, $n$ is the number of evaluation points.

\subsection{Synthetic Sinusoidal Strain Field}

This section involves the use of synthetic images undergoing sinusoidal displacements with different values for the period, similar to what was reported in recent papers [11, 20-21]. Based on these studies, the spatial resolution is defined as the lowest period over which the displacement or strain gradients can be reproduced without significant loss of amplitude, which is defined by the analyst according to the measurement requirements.

The sinusoidal displacement applied in the $x$-direction to generated deformed images satisfies the following equation:

$$
u(x, y)=A \cdot \sin (2 \pi x / P)
$$

where, $A$ is the amplitude, and $P$ is the period.

Thus, a set of eight sinusoidal deformed images were generated with periods varying from 120 to 400 pixels. For each simulation, the amplitude $A$ in Eq. (14) was adjusted in order to obtain a maximum peak of $1.2 \%$ in strain response. Fig. 13 shows an example of a pre-assigned sinusoidal strain field.

The deformed images were processed using both DIC algorithms. The VIC-2D software was tested using two different parameter settings. The first configuration (VIC-2D \#1) uses a subset size of 21 pixels; step size of 2 pixels; and strain window of 11 . The second configuration (VIC-2D \#2) uses a subset size of 41 pixels; step size of 2 pixels; and strain window of 21. In the SIFT-Meshless method, the 2-D analysis adopted two configurations corresponding to two different values of the radius of domain: $d_{i}=30$ pixels (SIFT-M \#1) and $d_{i}=40$ pixels (SIFT-M \#2). In this case, since it is a numerical experiment and noise was not involved, the test function showed in Fig. $4 \mathrm{~b}$ was modified for values of $\alpha_{s}$ varying from 2 to 3. Thus, by using Eq. (4), the minimum influence domain sizes are 60 pixels and 80 pixels for the first configuration $\left(d_{i}=30\right)$ and the second configuration $\left(d_{i}=40\right)$, respectively.

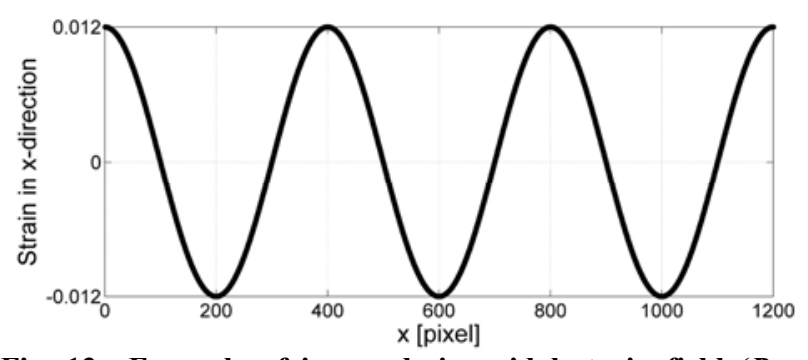

Fig. 13 Example of imposed sinusoidal strain field $(P=$ 400 pixels) used in the numerical tests. 

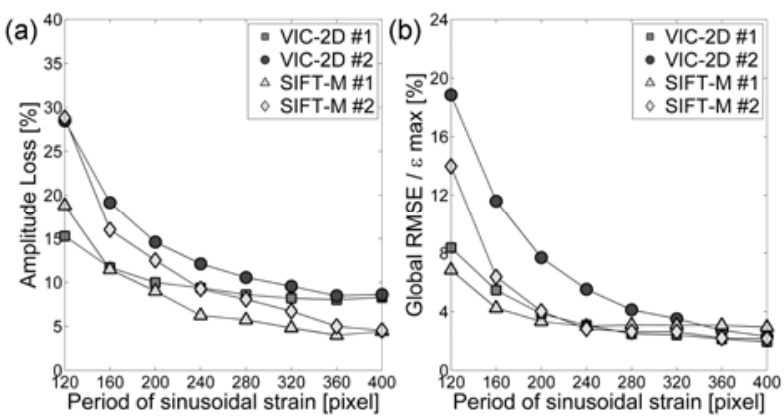

Fig. 14 Results obtained from synthetic sinusoidal gradient analysis.

Figs. 14a and 14b show the percentage of amplitude loss to reach the strain peak (maximum and minimum values) for the imposed sinusoidal strain field and the RMSE of the computed strain field using the two DIC techniques. From these figures, it can be seen that the SIFT-Meshless method was able to reproduce sinusoidal strains of periods up to 200 pixels with values of amplitude loss less than $10 \%$. As expected, more accurate results are obtained with the minimum radius of domain when the values of the period decreased, i.e. more heterogeneous field. In the same manner, the selection of a small virtual strain gage [22] size in the DIC analysis clearly improved the accuracy of the solution.

\subsection{Synthetic Gradient from a Closed-form Solution for the Stress Concentration around a Notch}

This section aimed to use a closed-form solution for stress concentration around notches to generate synthetic images in order to evaluate the accuracy and the spatial resolution of traditional and non-traditional DIC algorithms. For that, a displacement function derived from the Kirsch's solution [23] was formulated. The Kirsch's solution is a well-know analytical solution used to describe the strain distribution around a small circular hole in an infinite plate subjected to uniaxial tensile loads. Therefore, a more suitable error assessment can be performed in order to compare the performance of the DIC algorithms by simulating the actual strain gradient that develops around a notch.

The strain distribution from the Kirsh's solution plotted in Fig. 15 shows that the maximum strain at the notch boundary is three times higher than the nominal strain far away from the hole. Moreover, it can be seen that at a distance of half the notch radius, the strain decays to approximately half of its maximum value, named here as half-radius axial decay. This behavior is also observed in strain distributions around notches subjected to uniaxial tensile loading (see experimental results of the Section 4). Therefore, this characteristic can be used as control parameter to obtain different configurations of strain gradients to be simulated.

For simplicity, the Kirsch's equations were first rewritten to apply a displacement only along the $x$-direction, as shown in Fig. 16. Hence, the horizontal displacement was defined as

$$
u(x, y)=\frac{\varepsilon}{6}\left[2 x-\frac{R^{2}}{x}-\frac{R^{4}}{x^{3}}\right]
$$

where, $\varepsilon$ is the maximum strain at the notch boundary, and $\mathrm{R}$ is the notch radius in pixels.

In this analysis, a set of seven deformed images were simulated with maximum strain value of $0.7 \%$ ( $\varepsilon$ $=0.007)$ and values of radius $(\mathrm{R})$ varying from 200 to 500 pixels, corresponding to a minimum half-radius axial decay of 100 pixels and maximum of 250 pixels, respectively.

The VIC-2D analysis adopted two different parameter settings. The first configuration uses a subset size of 21 pixels; step size of 5 pixels; and strain window of 11 .

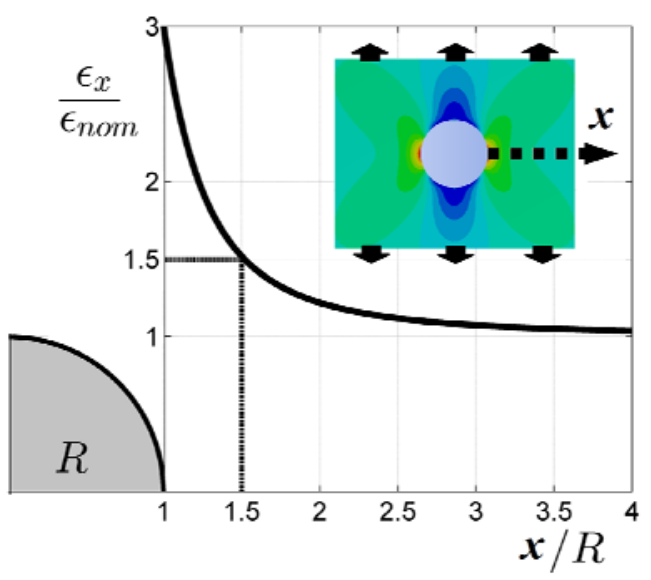

Fig. 15 Strain distribution $\varepsilon_{x x}$ along the horizontal axis from the Kirsch's solution. 


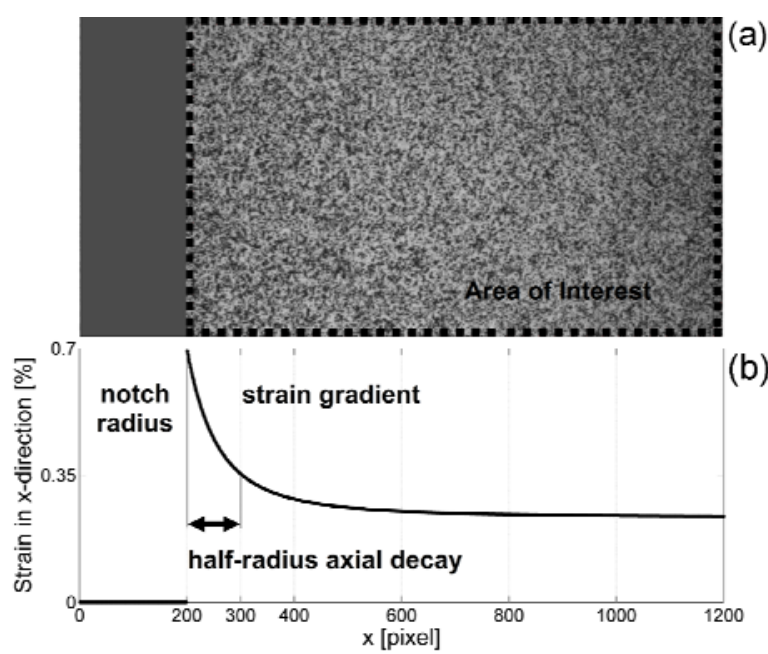

Fig. 16 (a) Area of interest used for validation (b) example of imposed strain field $(\varepsilon=0.007, R=200$ pixels, half-radius axial decay $=100$ pixels) used in the numerical tests.
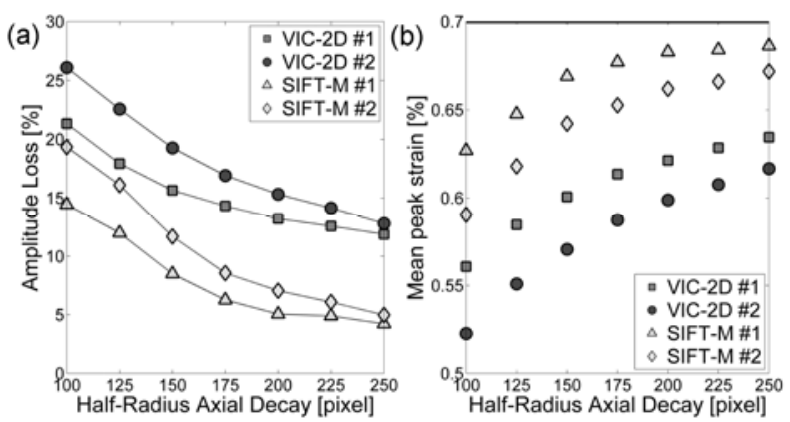

Fig. 17 Results obtained from synthetic gradient analysis with closed-form solution.

The second configuration uses a subset size of 41 pixels; step size of 5 pixels; and strain window of 21. The SIFT-Meshless analysis adopted two different values for the radius of domain: $d_{i}=80$ pixels and $d_{i}=$ 100 pixels. Similar to the subsection 6.1 the values of $\alpha_{s}$ vary from 2 to 3 . Thus, by using Eq. (4), the minimum influence domain sizes are 160 pixels and 200 pixels for the first configuration $\left(d_{i}=80\right)$ and the second configuration $\left(d_{i}=100\right)$, respectively.

The region outside of the area of interest shown in Fig. 16.a simulates the presence of a notch; therefore, the results obtained from this analysis shown in Fig. 17 take into consideration the error to capture the strain value at the notch boundary. For the SIFT-Meshless method, it can be seen that values of amplitude loss below of $10 \%$ are expected for values of half-radius axial decay above 150 pixels. These results confirm the good performance of the method to obtain reliable strain measurements near the notch boundary. In the other hand, the VIC-2D algorithm presented values of amplitude loss above of $10 \%$ for all the simulations. It is worth mentioning that the maximum values were extracted directly from the solution, and no extrapolation was used to estimate the maximum value at boundary of the simulated notch.

\subsection{Discussion}

In this study, two different metrics for evaluating the spatial resolution of the non-traditional DIC algorithm have been tested: synthetic sinusoidal strain field and synthetic strain gradient from a closed-form solution.

The first metric, proposed in Ref. [11], was focused on the amplitude loss to capture the maximum strain in a highly heterogeneous strain field. The control parameter is the period, $\mathrm{P}$, of the sinusoidal function. The numerical results shown in Fig. 14 confirmed that the SIFT-Meshless algorithm is able to perform high strain gradient measurements using an adequate experimental configuration.

The second metric, proposed in this paper, was focused on the amplitude loss to capture the maximum strain in the region surrounding a notch tip. The control parameter for this approach is the half-radius axial decay, which establishes that at a distance from the notch root equal to half the notch radius, the strain decreases to approximately half the maximum value reached. The numerical results shown in Fig. 17 confirmed the capability of the SIFT-Meshless algorithm to obtain accurate measurements along to the notch boundary. As shown in Fig. 18, since features points are detected over the notch boundary, strain measurements are possible to be determined along this critical area using the SIFT-Meshless method. This is in contrast to the traditional subset-based DIC algorithm that showed a limitation in obtaining the strain information at this critical area. This limitation happens because the point of measurement in subset-based 


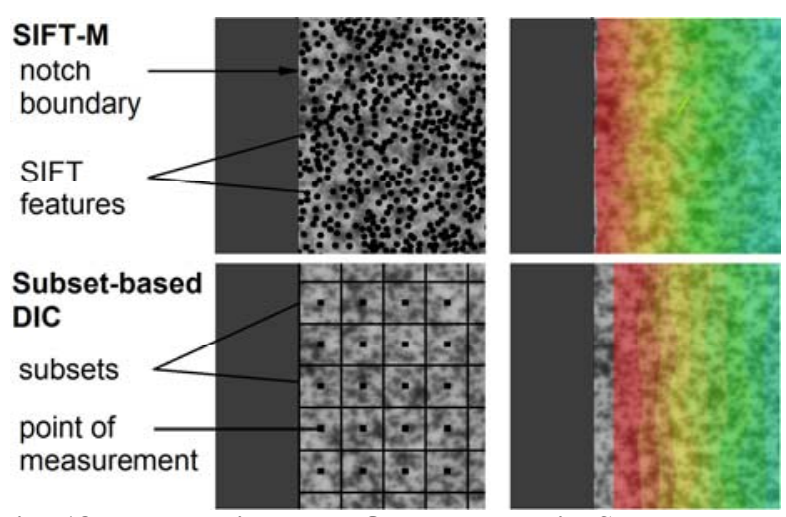

Fig. 18 Data points at ROI boundary in SIFT-Meshless method and traditional subset-based DIC.

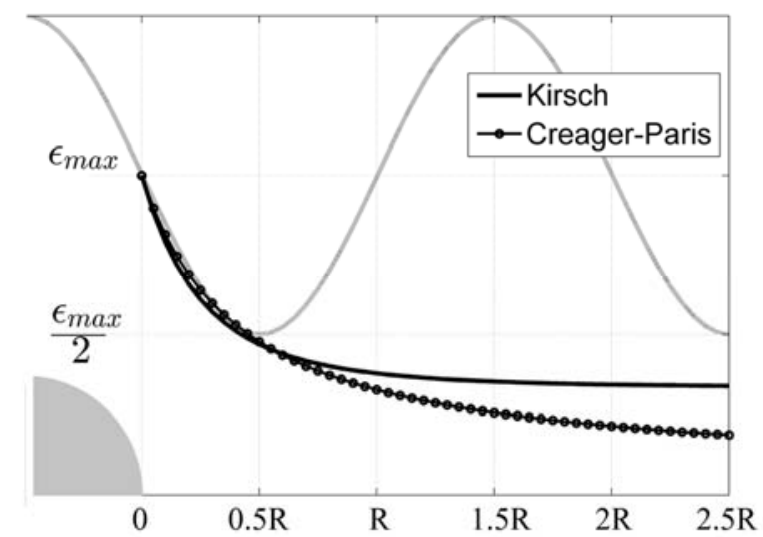

Fig. 19 Closed form solutions fitted by a sinusoidal function.

methods is located at the center of the subset; therefore, a data point is obtained at least half the subset away from the ROI boundary. It reflects in the error value to find the maximum strain values, as shown in the numerical and experimental results.

In addition, the half-radius axial decay can be also related to the pixel resolution needed to perform a measurement with a prescribed accuracy. For example, based on the results shown in Fig. 17a, values of amplitude loss of about 5\% and 15\% are expected for the SIFT-Meshless method and the VIC-2D software, respectively, when the half-radius axial decay is 200 pixels and the maximum expected strain is $7,000 \mu \varepsilon$. If the radius of the notch to be analyzed is equal to 1 $\mathrm{mm}$ (PC-2 specimen), i.e., $\mathrm{R}=2$-half-radius axial decay $=400$ pixels, then the pixel resolution required is $2.5 \mu \mathrm{m} / \mathrm{pixel}$. This example can be compared with the PC-2 specimen case (Section 4): a plate subjected to tensile stress with notch radius of $1 \mathrm{~m}$, at which a pixel resolution of approximately $2.5 \mu \mathrm{m} /$ pixel was used. From the experimental results shown in Fig. 6b, the values of loss amplitude obtained for the maximum load applied to the specimen (i.e., $\mathrm{P}=60 \mathrm{~N}$ with maximum strain of approximately 7,000 $\mu \varepsilon$ at notch boundary) were $2 \%$ and $12 \%$ for SIFT-Meshless method and VIC-3D software, respectively. These results are in agreement with the predicted values in Fig. 17a. Therefore, it can be concluded that this approach can be used to evaluate the expected error in obtaining the maximum strain value using traditional and non-traditional DIC algorithms with a certain experimental configuration.

Finally, the half-radius axial decay can be also related to the period of the sinusoidal function as shown in Fig. 19. It is shown that, the strain gradient behavior from the Kirsch's solution (for describing stresses around a hole in an infinite plate) and from the Creager \& Paris equations [24] (for describing stresses in parabolic, elliptic, U and narrow V-shaped notches) can be approximated by a sine function, i.e., half-radius axial decay $=\mathrm{P} / 4$. Thus, a priori error estimation of an algorithm can be obtained if numerical experiments are simulated beforehand using a displacement sinusoidal function with period $\mathrm{P}=2 \cdot \mathrm{R}$.

\section{Conclusions}

The present work shows and compares by means of experimental and numerical tests, the ability of a recently developed method, the SIFT-Meshless method, to perform high strain gradient measurements around shallow and deep notches, even under conditions of significant plasticity. The experimentally determined full-field strain responses are in agreement with results determined from numerical finite element simulations and from a commercially available DIC software. The capability of the SIFT-Meshless method for obtaining reliable strain measurements at the notch boundary zone, where the maximum strain values were expected, was 
demonstrated. Elastic and plastic strain values experimental measured at the different notch shapes, as high as $0.7 \%$ and $0.5 \%$, respectively, with errors less than $3 \%$ confirmed the validity of the method. In addition, the performance of the both DIC tested algorithms in terms of spatial resolution were successfully checked and compared.

\section{Acknowledgements}

G.L.G. Gonzáles gratefully acknowledges the support of the CNPq-Conselho Nacional de Desenvolvimento Científico e Tecnológico, Brazil (reference 152795/2016-2).

\section{References}

[1] Bruck, H., McNeill, S., Sutton, M. A., and Peters III, W. 1989. "Digital Image Correlation Using Newton-Raphson Method of Partial Differential Correction." Experimental Mechanics 29 (3): 261-7.

[2] Pan, B., Qian, K., Xie, H., and Asundi, A. 2009. "Two-dimensional digital Image Correlation for In-Plane Displacement and Strain Measurement: A Review." Measurement Science and Technology 20 (6): 062001.

[3] Pan, B., and Li, K. 2011. "A Fast Digital Image Correlation Method for Deformation Measurement." Optics and Lasers in Engineering 49 (7): 841-7.

[4] Sutton, M. A., Orteu. J. J., and Schreier, H. 2009. "Image Correlation for Shape, Motion and Deformation Measurements: Basic Concepts, Theory and Applications." Springer Science \& Business Media.

[5] Lagattu, F., Brillaud, J., and Lafarie-Frenot, M.-C. 2004. "High Strain Gradient Measurements by Using Digital Image Correlation Technique." Materials Characterization 53 (1): 17-28.

[6] Qian, C., Harper, L., Turner, T., and Warrior, N. 2011. "Notched Behaviour of Discontinuous Carbon Fibre Composites: Comparison with Quasi-isotropic Non-crimp Fabric." Composites Part A: Applied Science and Manufacturing 42 (3): 293-302.

[7] Hwang, S.-F., and Wu, W.-J. 2012. "Deformation Measurement Around a High Strain-Gradient Region Using a Digital Image Correlation Method.” Journal of Mechanical Science and Technology 26 (10): 3169-75.

[8] Ashrafi, M., and Tuttle, M. E. 2015. "High Strain Gradient Measurements in Notched Laminated Composite Panels by Digital Image Correlation." Composite, Hybrid, and Multifunctional Materials 4: $75-81$.
[9] Sun, Y., Pang, J. H., Wong, C. K., and Su, F. 2005. "Finite Element Formulation for a Digital Image Correlation Method." Applied Optics 44 (34): 7357-63.

[10] Pan, B., Wang, Z., and Lu, Z. 2010. "Genuine Full-field Deformation Measurement of an Object with Complex Shape Using Reliability-Guided Digital Image Correlation." Optics Express 18 (2): 1011-23.

[11] Wittevrongel, L., Lava, P., Lomov, S., and Debruyne, D. 2014. "A self Adaptive Global Digital Image Correlation Algorithm.” Experimental Mechanics 55 (2): 361-78.

[12] Zhu, R., Xie, H., Hu, Z., Jiang, L., Guo, B., and Li, C. 2015. "Performances of Different Subset Shapes and Control Points in Subset-based Digital Image Correlation and Their Applications in Boundary Deformation Measurement." Applied Optics 54 (6): 1290-301.

[13] Gonzáles, G., and Meggiolaro, M. 2015. "Strain Field Measurements Around Notches Using SIFT Features and Meshless Methods." Applied Optics 54 (14): 4520-8.

[14] Lowe, D. G. 1999. "Object Recognition from Local Scale-Invariant Features." The Proceedings of the Seventh IEEE International Conference 1150-7.

[15] Lowe, D. G. 2004. "Distinctive Image Features From Scale-Invariant Keypoints." International Journal of Computer Vision 60 (2): 91-110.

[16] Liu, G.-R., and Gu, Y.-T. 2005. An Introduction to Meshfree Methods and Their Programming. Springer.

[17] VIC-3D, ®Software. 2010. Correlated Solutions Inc. $\mathrm{http}: / / \mathrm{www}$. correlatedsolutions.com/.

[18] ISO, I., and Guide, I. 2007. International Vocabulary of Metrology-Basic and General Concepts and Associated Terms.

[19] Belytschko, T., Lu, Y. Y., and Gu, L. 1994. "Element-Free Galerkin Methods." International Journal for Numerical Methods in Engineering 37 (2): 229-56.

[20] Bornert, M., Brémand, F., Doumalin, P., Dupré, J.-C., Fazzini, M., Grédiac, M., Hild, F., Mistou, S., Molimard, J., and Orteu, J.-J. 2009. “Assessment of Digital Image Correlation Measurement Errors: Methodology and Results." Experimental Mechanics 49 (3): 353-70.

[21] Pan, B., Wang, B., Lubineau, G., and Moussawi, A. 2015. "Comparison of Subset-Based Local and Finite Element-based Global Digital Image Correlation." Experimental Mechanics 55 (5): 887-901.

[22] Reu, P. 2015. "Virtual Strain Gage Size Study." Experimental Techniques 39 (5): 1-3.

[23] Shukla, A., and Dally, J. W. 2010. "Experimental Solid Mechanics." College House Enterprises Knoxville.

[24] Creager, M., and Paris, P. C. 1967. "Elastic Field Equations for Blunt Cracks with Reference to Stress Corrosion Cracking." International Journal of Fracture Mechanics 3 (4): 247-52. 